\title{
A satellite-based method for modeling ionospheric slant TEC from GNSS observations: algorithm and validation
}

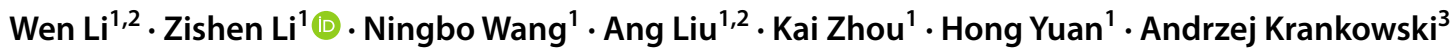

Received: 8 June 2020 / Accepted: 6 October 2021 / Published online: 15 November 2021

(c) The Author(s) 2021

\begin{abstract}
Total Electron Content (TEC) modeling is critical for Global Navigation Satellite System (GNSS) users to mitigate ionospheric delay errors. The mapping function is usually used for Vertical TEC ionospheric correction models for slant and vertical TEC conversion. But the mapping function cannot characterize TEC variation in different azimuths between the user and satellites. The ionospheric modeling error resulting from the mapping function tends to be bigger in middle and low latitudes. Therefore, a new algorithm for ionospheric Slant TEC (STEC) modeling with Satellite-based Ionospheric Model (SIM) is proposed in this contribution. Validation tests are carried out with GNSS observation data from the Crustal Movement Observation Network of China during different solar activities and in different seasons. The performance of SIM is compared with that of several commonly-used Global Ionospheric Map (GIM) and Regional Ionospheric Map (RIM) products. The results show that the STEC bias and STD of SIM are within 1.0 TECU and about 2.0 TECU, respectively, and SIM can correct over $90 \%$ STEC RMS errors, outperforming the GIM and RIM products. Consequently, the SIM algorithm can be a new option for high-accuracy ionospheric delay correction in regional and local GNSS networks.
\end{abstract}

Keywords GNSS $\cdot$ STEC modeling $\cdot$ SIM $\cdot$ GIM $\cdot$ RIM

\section{Introduction}

As a major error source in a Global Navigation Satellite System (GNSS), the correction accuracy of ionospheric delay directly affects the availability, accuracy, reliability, and integrity of GNSS services. The development of rapid and real-time high-accuracy GNSS applications has become a research focus in recent years (Juan et al. 2012; Shi et al. 2012). This trend leads to the ever-growing demand for highly accurate and efficient ionospheric correction methods. Such corrections can help dual-frequency users obtain

Zishen Li

lizishen@aircas.ac.cn

1 Aerospace Information Research Institute (AIR), Chinese Academy of Sciences (CAS), No.9 Dengzhuang South Road, Haidian District, Beijing 100094, China

2 School of Electronic, Electrical and Communicating Engineering, University of Chinese Academy of Sciences (UCAS), No.19 Yuquan Road, Shijingshan District, Beijing 100049, China

3 Space Radio, Diagnostics Research Centre, University of Warmia and Mazury in Olsztyn, 10-720 Olsztyn, Poland fixed solutions and rapid convergence in Precise Point Positioning (PPP) applications (Geng et al. 2010; Wang et al. 2019).

Ionospheric correction models such as broadcast models provided by major GNSS systems (IS-GPS 2004; Yang et al. 2020; Montenbruck and González 2019), models using ground-based observation data for GNSS augmentation purposes (Li et al. 2020), and single-station ionospheric models (Yasyukevich et al. 2020) are used to mitigate ionospheric delay errors. All these common models are ionospheric Vertical TEC (VTEC) models. Many mathematical functions have been used for VTEC representation on global and regional scales, and there is much research on their performance analysis and comparison (Santis et al. 1999; Li et al. 2019). However, with an increasing number of densely distributed GNSS reference stations, it is found that the accuracy of different ionospheric VTEC modeling functions is basically equivalent when the elevation mask is 15 degrees and above (Li et al. 2014a).

We think that two factors mainly restrict the accuracy improvement of ionospheric VTEC correction models. One is the mapping function. Although many mapping functions have been developed (Feltens 1998; Schaer 1999; Klobuchar 
1987), the errors generally become larger with lower elevation angles. All these mapping functions are basically equivalent when the elevation angle is above 30 degrees. Moreover, the mapping functions are only elevation-dependent and totally ignore horizontal gradients in ionospheric TEC variation (Schaer 1999). The isotropic hypothesis of TEC distribution along different azimuths at the Ionospheric Pierce Point (IPP) may be reasonable in regions with moderate ionospheric activities. But for regions with a high level of ionospheric activities, STEC/VTEC conversion errors will be significantly increased, and the accuracy of VTEC models will be greatly reduced (Lanyi and Roth 1988; Conker and El-Arini 2002). The other factor is model establishment mode. Ionospheric VTEC models are established for all GNSS satellites taken as a whole and can only provide an averaged solution. But this is not the optimal solution for each individual satellite.

Therefore, we present a satellite-based method for direct ionospheric STEC modeling and correction. This algorithm is well applicable to regional and local GNSS networks within several hundred kilometers. Based on descriptions of motivation and physics fundamental behind the algorithm, detailed processing procedures are given in the methodology. Validation experiments are carried out for regional GNSS networks with different inter-station distances and under different levels of solar activities. Several commonlyused GIM and RIM products are also used for inter-comparison with the correction accuracy of the proposed algorithm.

\section{Methodology}

We focus on direct STEC modeling to avoid modeling errors due to the mapping function. Currently, studies on ionospheric STEC correction models are very few. Sparks et al. (2004) have suggested a conical domain approach for STEC delay computation without using conventional gridbased estimations. But a thin-shell obliquity factor is still needed in the conical model of each satellite. Our goal is to get rid of the obliquity factor and STEC/VTEC conversion mode. Although STEC variation along the propagation paths between GNSS user and different satellites can be rather complicated, the STEC values observed from one satellite on a continuous observation arc are usually quite smooth for a network within dozens to hundreds of kilometers. Therefore, ionospheric STEC delay can be modeled and corrected satellite-by-satellite with Satellite-based Ionospheric Model (SIM) (Li et al. 2014a) established for every single satellite in a regional or local GNSS network. As ionospheric TEC is highly homogenous and correlated for a small coverage in a short period, a low-order polynomial function can be adopted for STEC modeling. In general, this assumption is reasonable for small-scale networks, and the performance of the STEC SIM method would deteriorate with increasing inter-station distance. Moreover, the temporal resolution of SIM corrections should also be an optimal option for both algorithm performance and computation complexity. For a specific satellite, the major procedures involved in establishing and applying the STEC SIM method are described.

First, we use the geometry-free combination of dualfrequency GNSS observation data to determine raw ionospheric STEC measurement along line-of-sight between GNSS satellite and user. The code smoothing approach is adopted during each continuous observation arc to obtain high-precision ionospheric TEC measurements ( $\mathrm{Li}$ et al. 2014b).

Second, we remove satellite and receiver Differential Code Bias (DCB) from raw STEC measurement to obtain accurate STEC observable (Yasyukevich et al., 2020; Zhang et al., 2017). Much previous research has studied DCB estimation methods and variation characteristics (Mylnikova et al. 2015; Yasyukevich et al. 2015). Although multi-GNSS satellite DCBs have been provided by some multi-GNSS experiment (MGEX) analysis centers like the Chinese Academy of Sciences (CAS), the receiver DCBs of the Crustal Movement Observation Network of China (CMONOC) stations are not publicly available in the following experiments. In this research, satellite DCBs are fixed to CAS MGEX DCB solutions and receiver DCBs are estimated using the IGGDCB method (Li et al. 2012). It should be mentioned that the mapping function is used during CAS DCB product generation. But as an elevation angle-dependent weighting strategy is adopted to reduce the influence of low elevation angles, such modeling error can be considered as very small. The one-day multi-GNSS DCB solution is computed using 24-h observation arc with a latency of one day and few hours for real-time applications. Furthermore, in order to improve DCB estimates stability in case of real-time data stream interruptions, an automatic bias realignment procedure is adopted to generate the three-day mean value of satellite and receiver DCBs. The three-day bias solution is then used to correct satellite and receiver DCBs and to generate biasfree slant ionospheric delays for subsequent TEC modeling. According to long-term performance monitoring and evaluation of DCB products from CAS and other major sources, such as CODE and DLR, results show equivalent precision levels and good consistency between these DCB solutions (Wang et al. 2016, 2020). Therefore, the impact of different DCB product sources on the performance of the proposed algorithm should be very limited.

Third, we estimate and generate STEC SIM corrections. Equation (1) shows the polynomial function with STEC measurements from reference stations during a resolving interval. The SIM corrections include IPP geometric center coordinates $\left(\varphi_{0}, \lambda_{0}\right)$ and the polynomial coefficients $E_{i j} i=0, \ldots, n \mathrm{j}=0, \ldots, \mathrm{m} . \varphi_{0}$ and $\lambda_{0}$ are dependent on 
latitudes and longitudes of all observed IPP points $\left(\varphi_{k}, \lambda_{k}\right)$ $k=1,2, \ldots \ldots, N$ within the specific time window and can be calculated as $\varphi_{0}=\frac{1}{N} \sum_{k=1}^{k=N} \varphi_{k}$ and $\lambda_{0}=\frac{1}{N} \sum_{k=1}^{k=N} \lambda_{k}$, respectively. The polynomial coefficients can be estimated with the Weighted Least Squares (WLS) method and an elevationdependent weight matrix.

$$
\begin{gathered}
\operatorname{STEC}_{k}^{s}=\sum_{i=0}^{n} \sum_{j=0}^{m} E_{i j}\left(\varphi_{k}-\varphi_{0}\right)^{i}\left(\lambda_{k}-\lambda_{0}\right)^{j} \\
k=1,2, \ldots \ldots, N
\end{gathered}
$$

where $\mathrm{STEC}_{k}^{s}$ is the observable of satellite $s$ at epoch $k, \varphi_{k}$ and $\lambda_{k}$ are IPP latitude and longitude of $\mathrm{STEC}_{k}^{s}$, respectively, $N$ is the number of observation epochs for satellite $s$ within the modeling interval, $\varphi_{0}$ and $\lambda_{0}$ are latitude and longitude of the geometric center of all IPPs within the modeling interval, $n$ and $m$ are the latitudinal and longitudinal polynomial fitting order, respectively, $E_{i j} i=0, \ldots, n \mathrm{j}=0, \ldots, \mathrm{m}$ are the unknown polynomial coefficients to be estimated.

In WLS processing, the observation equation can be written as below.

$A_{N \times \text { count_ } X} X_{\text {count } \_X \times 1}=B_{N \times 1}$

where $N$ equals the number of observation epochs, $B$ is the STEC observables vector as $\mathrm{B}_{\mathrm{N} \times 1}=\left[T E C_{1} \ldots T E C_{i} \cdots T E C_{\mathrm{N}}\right]^{T}$, count $\_X$ is the number of polynomial coefficients to be estimated and equals count $\_X=(n+1) \cdot(m+1), \mathrm{X}$ is the unknown polynomial parameters vector as $X_{\text {count }-X \times 1}=\left[E_{00}, \ldots, E_{i j}\right]^{T}, A$ is the observation coefficients matrix as shown in the following equation.
Then, the weight function is the sinusoidal value of GNSS elevation angle at each observation epoch and shown as below.

$P_{N \times N}=\operatorname{diag}\left(\sin \theta_{1} \cdots \sin \theta_{i} \cdots \sin \theta_{N}\right)$

where $P_{\mathrm{N} \times \mathrm{N}}$ is diagonal weight matrix, $\theta_{\mathrm{k}} k=1,2, \ldots \ldots, N$ is corresponding elevation angle for $\operatorname{STEC}_{k} k=1,2, \ldots \ldots, N$.

Finally, for ionospheric delay correction at the user receiver, the SIM corrections can be matched according to the satellite PRN number, the product temporal resolution, and user observation epoch. The STEC value for a given observation epoch can be directly calculated by Eq. (1) with the corresponding user's IPP location and SIM corrections.

\section{Validation experiments}

To evaluate the performance of the proposed ionospheric STEC SIM method, validation experiments are carried out under different levels of ionospheric activities and in the middle and low latitudes of China through comparison with several conventional ionospheric VTEC correction models. The experiments are carried out in real-time processing mode.

\section{Data description}

The GNSS observation data are collected by CMONOC with a sampling interval of $30 \mathrm{~s}$. Day of Year (DOY) 61 to 75 of the year 2014, 2016 and 2018 are selected as three typical experimental periods under high, medium and low solar

$A_{N \times \text { count } \_}=\left(\begin{array}{cccccc}1 & \lambda-\lambda_{0} & \varphi-\varphi_{0} & \left(\varphi-\varphi_{0}\right)\left(\lambda-\lambda_{0}\right) & \left(\varphi-\varphi_{0}\right)^{2} & \left(\varphi-\varphi_{0}\right)^{2}\left(\lambda-\lambda_{0}\right) \\ \vdots & \vdots & \vdots & \vdots & \vdots & \vdots \\ 1 & \lambda_{N}-\lambda_{0} & \varphi_{N}-\varphi_{0} & \left(\varphi_{N}-\varphi_{0}\right)\left(\lambda_{N}-\lambda_{0}\right) & \left(\varphi_{N}-\varphi_{0}\right)^{2} & \left(\varphi_{N}-\varphi_{0}\right)^{2}\left(\lambda_{N}-\lambda_{0}\right)\end{array}\right)$

Fig. 1 SSN time series of different experimental periods

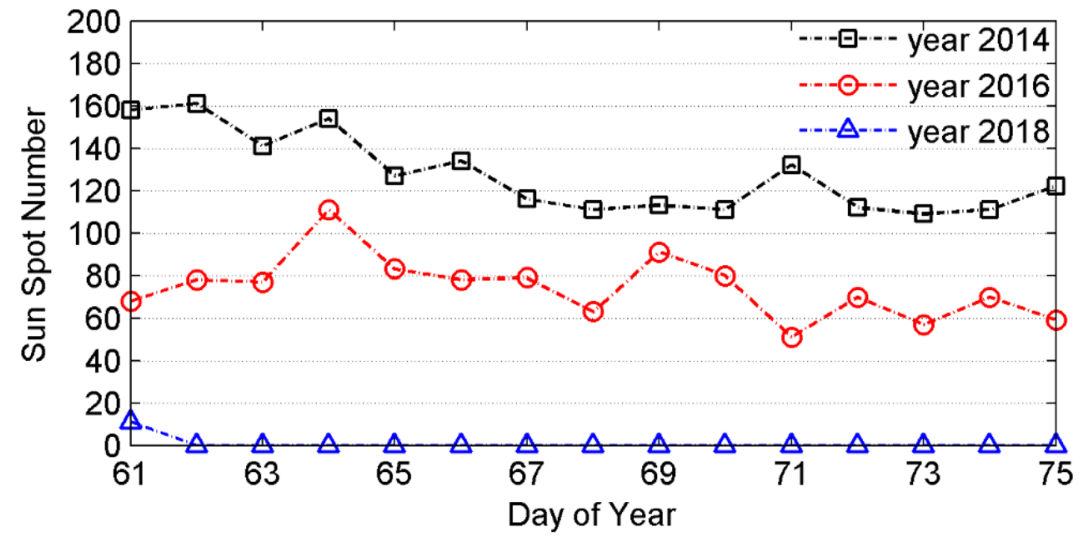




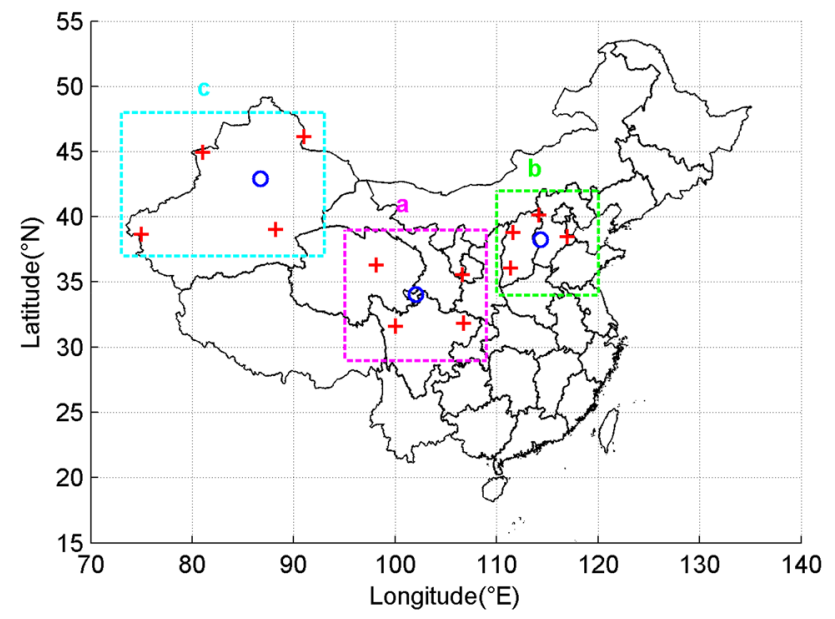

Fig. 2 Distribution of reference (red cross) and user (blue circle) stations for experimental network (a), (b) and (c) (marked by dashed rectangular)

activities, respectively, as illustrated in Fig. 1 with OMNIWeb data from NASA (https://omniweb.gsfc.nasa.gov/ index.html). Additionally, DOY 61-75, 165-179, 259-273 and 349-363 of 2014 are chosen as four representative test periods around the spring equinox, summer solstice, autumn equinox and winter solstice, respectively, for performance test during different seasons. The SIM algorithm is separately implemented in three regional GNSS networks. Figure 2 shows the layout of each experimental network, with more details being provided in Table 1 . The reference stations basically define the boundary of the target service area and the expected effective range of the SIM corrections. The user should use the corresponding SIM products of the network it belongs to. Due to a lack of raw observation data, the SIM corrections are unavailable for network (a) on DOY 61, 62, and 69 of 2014, network (b) on DOY 62 of 2014 and network (c) on DOY 62 of 2014 and DOY 73-75 of 2016.

\section{Processing strategy}

The SIM algorithm is realized for 32 GPS satellites independently with a cut-off elevation angle of 15 degrees. SIM corrections are generated with STEC measurements from the previous 5-min observation arc and updated every minute using a sliding time window for each satellite. As ionospheric TEC gradient is bigger in the latitudinal than longitudinal direction, STEC polynomial fitting order is set as 2 and 1, respectively, that is $n=2$ and $m=1$ in (1). Therefore, a set of eight parameters is provided during each resolving interval, including two IPP geometric center coordinate parameters and six polynomial coefficients. These corrections are used for direct STEC correction on the user stations.

For comparison with VTEC correction models, three common products are utilized, including UQRG-rapid GIM provided by Universitat Politècnica de Catalunya (UPC) (Hernández-Pajares et al. 2009, 2017), IOSR—daily RIM of China provided by China Earthquake Administration (CEA) and CRTC - real-time RIM of China provided by Chinese Academy of Sciences (CAS). Their major characteristics are listed in Table 2.

On the user station, STEC measurement extracted from dual-frequency GNSS data with carrier-to-code leveling and
Table 1 Detailed information of experimental networks

\begin{tabular}{|c|c|c|c|c|c|c|c|c|}
\hline \multirow[t]{3}{*}{ Network } & \multirow[t]{3}{*}{ User station } & \multirow[t]{3}{*}{ Reference stations } & \multicolumn{6}{|c|}{ Inter-station distance (in $\mathrm{km}$ ) } \\
\hline & & & \multicolumn{3}{|c|}{ Reference-reference } & \multicolumn{3}{|c|}{ Reference-user } \\
\hline & & & Min & $\operatorname{Max}$ & AVE & Min & Max & AVE \\
\hline $\mathrm{a}$ & GSMA & $\begin{array}{l}\text { QHDL GSPL } \\
\text { SCGZ SCBZ }\end{array}$ & 410 & 767 & 645 & 327 & 499 & 428 \\
\hline $\mathrm{b}$ & HELQ & $\begin{array}{l}\text { HEYY HECX } \\
\text { SXKL SXLF }\end{array}$ & 302 & 558 & 366 & 209 & 353 & 259 \\
\hline $\mathrm{c}$ & XJWL & $\begin{array}{l}\text { XJWQ XJQH } \\
\text { XJBL XJRQ }\end{array}$ & 787 & 1140 & 884 & 449 & 1091 & 636 \\
\hline
\end{tabular}

Table 2 Major characteristics of different ionospheric models

\begin{tabular}{|c|c|c|c|c|c|c|}
\hline \multirow[t]{2}{*}{ Name } & \multirow[t]{2}{*}{$\begin{array}{l}\text { Temporal } \\
\text { resolution }\end{array}$} & \multicolumn{2}{|l|}{ Spatial coverage } & \multicolumn{2}{|c|}{$\begin{array}{l}\text { Spatial resolu- } \\
\text { tion }\end{array}$} & \multirow{2}{*}{$\begin{array}{l}\text { Number of refer- } \\
\text { ence stations in } \\
\text { China }\end{array}$} \\
\hline & & Lat & Lon & Lat & Lon & \\
\hline SIM & $5 \mathrm{~min}$ & & & & & \\
\hline UQRG & $15 \min$ & $87.5^{\circ} \mathrm{S}-87.5^{\circ} \mathrm{N}$ & $180^{\circ} \mathrm{W}-180^{\circ} \mathrm{E}$ & $2.5^{\circ}$ & $5^{\circ}$ & $10-15$ \\
\hline IOSR & $2 \mathrm{~h}$ & $15^{\circ} \mathrm{N}-55^{\circ} \mathrm{N}$ & $70^{\circ} \mathrm{E}-140^{\circ} \mathrm{E}$ & $1^{\circ}$ & $1^{\circ}$ & 260 \\
\hline CRTC & $15 \mathrm{~min}$ & $15^{\circ} \mathrm{N}-55^{\circ} \mathrm{N}$ & $70^{\circ} \mathrm{E}-140^{\circ} \mathrm{E}$ & $1^{\circ}$ & $1^{\circ}$ & 80 \\
\hline
\end{tabular}


DCB calibration is considered as the 'true' value of line-ofsight ionospheric delay and denoted as STEC $_{\text {obs. }}$. The STEC estimation obtained with a correction model is denoted as STEC $_{\text {model, }}$ and several indicators used as a measure of ionospheric correction accuracy are defined as

$\mathrm{STEC}_{\mathrm{res}}=\mathrm{STEC}_{\text {model }}-\mathrm{STEC}_{\mathrm{obs}}$

$\mathrm{STEC}_{\mathrm{bias}}=\sum_{i=1}^{n}\left(\mathrm{STEC}_{\mathrm{res}}\right)_{i} / n$

$\mathrm{STEC}_{\mathrm{std}}=\sqrt{\sum_{i=1}^{n}\left(\left(\mathrm{STEC}_{\mathrm{res}}\right)_{i}-\mathrm{STEC}_{\mathrm{bias}}\right)^{2} /(n-1)}$

$\mathrm{STEC}_{\mathrm{rel}}=\sqrt{\sum_{i=1}^{n}\left(\mathrm{STEC}_{\mathrm{res}}\right)_{i}^{2} / n} / \sqrt{\sum_{i=1}^{n}\left(\mathrm{STEC}_{\mathrm{obs}}\right)_{i}^{2} / n} \times 100 \%$

where $n$ is the number of observation epochs during a given time interval, STEC $_{\text {res }}$ is residual of STEC model, $_{\text {STEC }}$ bias is the mean value (BIAS) of $\mathrm{STEC}_{\mathrm{res}}, \mathrm{STEC}_{\text {std }}$ is the standard deviation (STD) of $\mathrm{STEC}_{\mathrm{res}}$, and $\mathrm{STEC}_{\mathrm{rel}}$ is relative RMS error (REL) of STEC $_{\text {res }}$.

\section{Results and discussion}

All validation experiments are summarized in Table 3. The performance of CRTC is tested under high and low solar activities but is temporarily not available during the test period in 2016. Detailed analysis of experiment results are given in this section. It should be noted the correction accuracy mentioned hereinafter refers to that of ionospheric STEC values.

\section{Model performance during different local time}

Since ionospheric TEC has diurnal variation characteristics, the performances of different ionospheric models within a day are studied and compared at first. Figure 3 shows the correction accuracy of each model during every local hour on DOY 61 of 2014, 2016 and 2018 at user station GSMA.

It can be seen from Fig. 3 that there is a systematic offset in STEC residuals of UQRG, IOSR, and CRTC, and the systematic errors grow bigger with higher solar activities. UQRG's

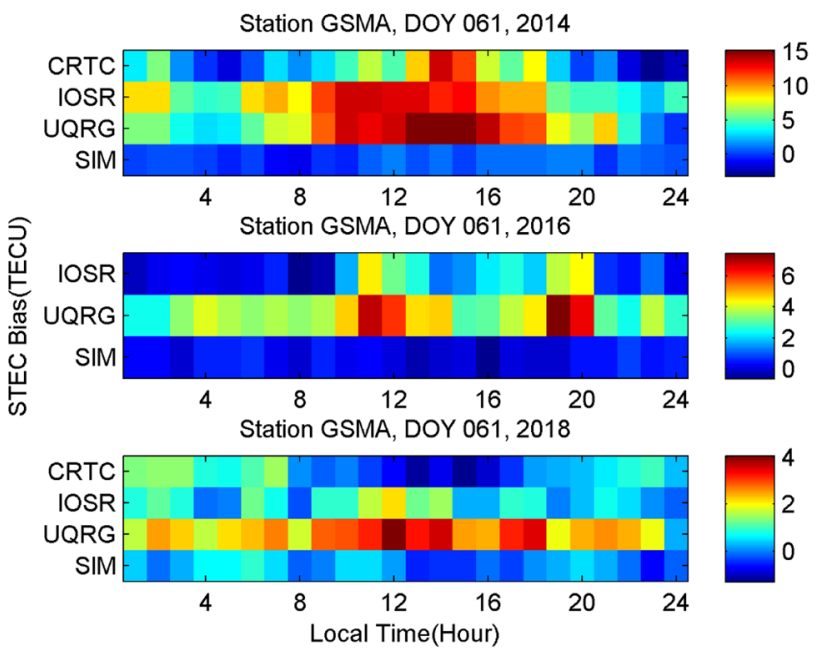

Fig. 3 Ionospheric hourly bias of different ionospheric models at station GSMA under different solar activities. DOY 61 of 2014 (top), DOY 61 of 2016 (middle), and DOY 61 of 2018 (bottom)

bias is larger than that of the RIM products, and this is mainly because a very limited number of IGS sites are used for its modeling within China. The hourly bias of CRTC is smaller than that of IOSR due to its higher temporal resolution. Additionally, the systematic offset is generally larger during the daytime than the night hours and tends to reach the daily maximum at noon. But the hourly bias of SIM is very small and without systematic error, suggesting the proposed algorithm can ideally reflect STEC variation throughout the day.

A similar intra-day variation pattern of model performance is also witnessed at station HELQ and XJWL. Figure 4 illustrates the hourly correction accuracy of each model, averaged among the three user stations on DOY 61, 2014, 2016, and 2018, respectively.

As shown in Fig. 4, the SIM bias is mostly smaller than $1 \mathrm{TECu}$, but the other products have a big systematic bias, especially under high solar activities. The hourly STD of the four models shows a decrease from 2014 to 2018 with a lower level of ionosphere activities. The SIM STD is also smaller than the other products, indicating smaller fluctuation of its STEC residuals and stable consistency between its model estimations and the local ionosphere. SIM can
Table 3 Overview of all validation experiments

\begin{tabular}{|c|c|c|c|c|c|}
\hline \multirow[t]{2}{*}{ User Station } & \multicolumn{2}{|c|}{ Test Period } & \multirow[t]{2}{*}{ Solar Activity } & \multirow[t]{2}{*}{ Season } & \multirow[t]{2}{*}{ Ionospheric Model } \\
\hline & DOY & Year & & & \\
\hline \multirow[t]{6}{*}{ GSMA HELQ XJWL } & $61-75$ & 2014 & High & Spring & SIM, UQRG, IOSR, CRTC \\
\hline & $165-179$ & & & Summer & \\
\hline & $259-273$ & & & Autumn & \\
\hline & $349-363$ & & & Winter & \\
\hline & $61-75$ & 2016 & Medium & Spring & SIM, UQRG, IOSR \\
\hline & $61-75$ & 2018 & Low & Spring & SIM, UQRG, IOSR, CRTC \\
\hline
\end{tabular}


Fig. 4 Average STEC hourly bias, STD, and relative RMS error on all user stations for each ionospheric model. Correction accuracy of SIM under different solar activities (top), of UQRG under different solar activities (second), of IOSR under different solar activities (third), and of CRTC under different solar activities (bottom)
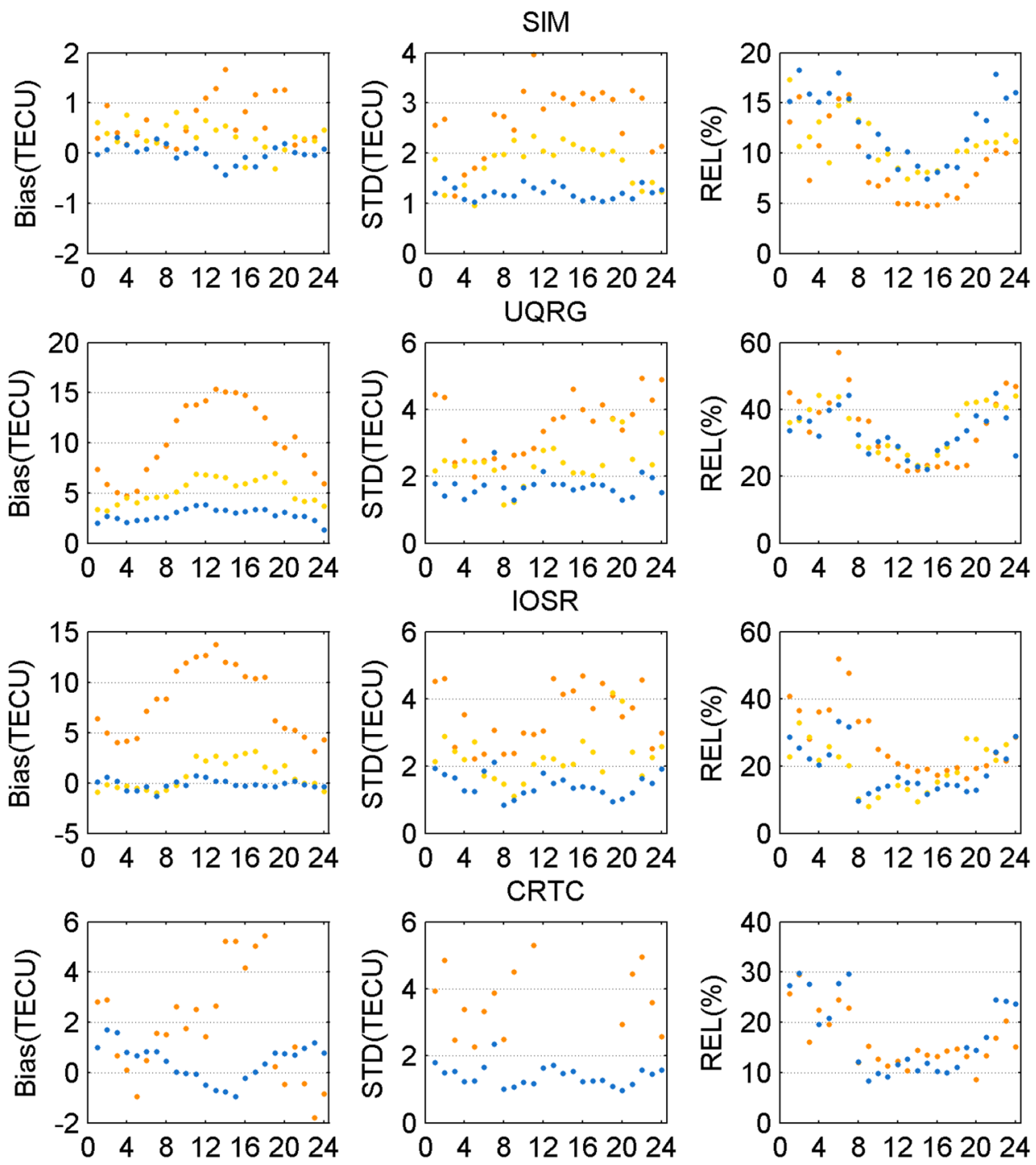

DOY 61 of 2014
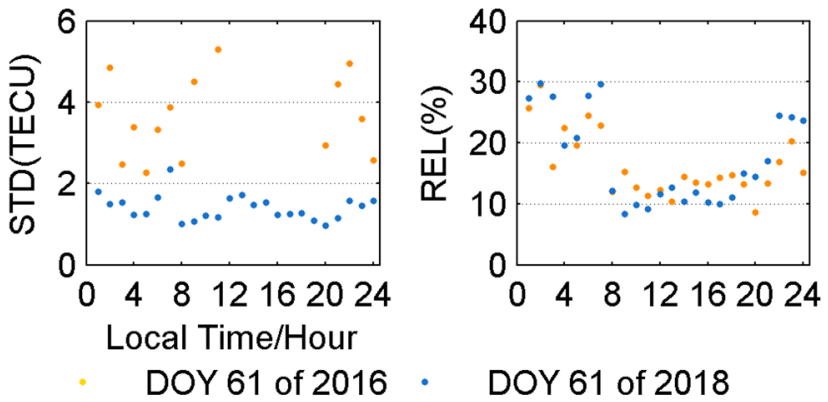

DOY 61 of 2018 correct about $85 \%$ to $95 \%$ STEC RMS errors within a single day and no less than $80 \%$ even in the worst cases. As a GIM product with very few reference stations in China, UQRG can correct about 60-80\% RMS errors. IOSR and CRTC can correct about $60-90 \%$ and $70-90 \%$ STEC RMS errors, respectively. It can be noted that the IOSR hourly bias is remarkably increased in 2014. This can be mainly attributed to the fact that the 2-h temporal resolution cannot adequately represent complicated TEC variation under high solar activities.

\section{Model daily performance}

Based on the study of intra-day characteristics of ionospheric correction performance, the STEC daily correction accuracy of the four ionospheric models will be analyzed and compared in this section. Taking into account of the impact of solar activities, we also select DOY 61 of 2014, 2016, and 2018 as the representative experimental days with high, medium, and low level of ionospheric activities, respectively.

\section{Daily correction accuracy for each satellite}

As SIM corrections are independently generated for different satellites, we first examine the daily performance of different models for each individual GPS satellite. Figure 5 shows STEC daily correction accuracy averaged among three experimental days with different ionospheric activities (DOY 61 of 2014, 2016, and 2018) for every GPS satellite at each user station.

We can see from Fig. 5 that the correction accuracy of the three GIM/RIM products show big difference between different satellites, but the performance of SIM is more stable for all the satellites. This phenomenon is in line with the characteristic of modeling methods for different 
Fig. 5 STEC daily bias, STD, and relative RMS error for each GPS satellite. Average correction accuracy of different solar activities at GSMA (top), HELQ (middle), and XJWL (bottom)
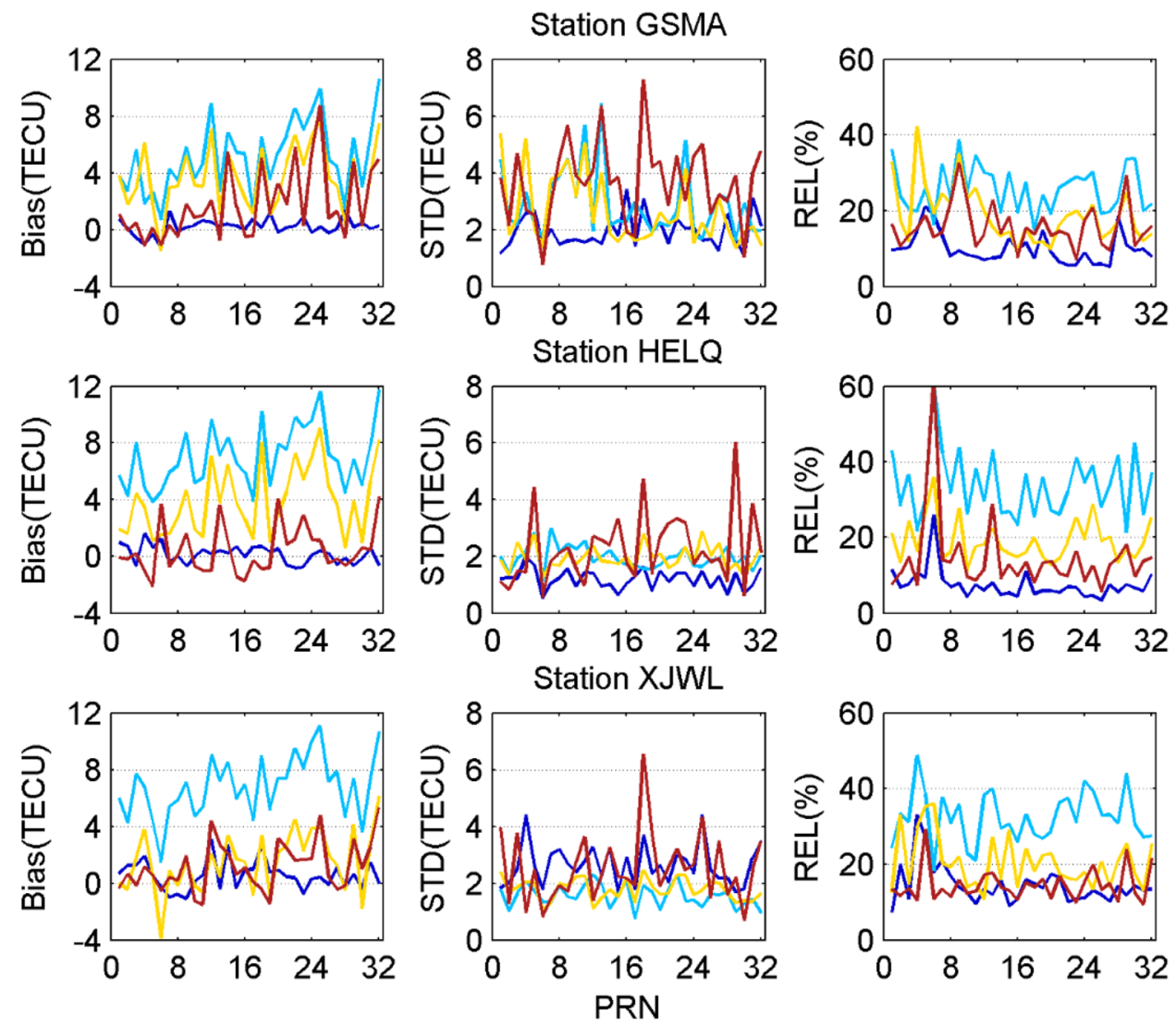

SIM

UQRG

IOSR correction models. For conventional GIM and RIM products, all ionospheric measurements are processed simultaneously to provide a unified solution for all satellites, and the model performance usually varies from satellite to satellite. But the SIM method can reflect TEC variation features of each satellite accurately to provide an individual optimal solution. In this way, the ionospheric correction accuracy can be improved. This is also one of the major motivations and advantages of the proposed algorithm.

\section{Daily correction accuracy for all satellites}

After analyzing the performance for a single satellite, the daily correction accuracy for the GPS system will be discussed as follows. Model daily performance for all GPS satellites at each user station is computed under different solar activities, and the average results among the three days are given in Table 4.

We can see in Table 4 that for the three GIM/RIM products, daily STD at GSMA is bigger than the other two stations. The reason may be that the ionospheric activities and TEC variation are more complicated over GSMA with lower latitude. But for SIM corrections, its accuracy at XJWL is slightly lower than other stations because of larger daily STD. This can be mainly attributed to the longer inter-station distance of regional network (network (c) in Fig. 2) than the other networks. Therefore, coverage range and inter-station distance are crucial factors in SIM
Table 4 Averaged daily model performance on DOY 61 of 2014, 2016 and 2018 for all GPS satellites. The unit for bias and STD is TECU, while relative RMS error (REL) is in percentile (\%)

\begin{tabular}{|c|c|c|c|c|c|c|c|c|c|c|c|c|}
\hline \multirow[t]{2}{*}{ Station } & \multicolumn{3}{|l|}{ SIM } & \multicolumn{3}{|c|}{ UQRG } & \multicolumn{3}{|c|}{ IOSR } & \multicolumn{3}{|c|}{ CRTC } \\
\hline & Bias & STD & REL & $\overline{B i a s}$ & STD & REL & Bias & STD & $\overline{\mathrm{REL}}$ & Bias & STD & REL \\
\hline GSMA & 0.23 & 1.81 & 9.20 & 4.85 & 2.72 & 24.36 & 3.36 & 2.46 & 17.43 & 1.74 & 3.47 & 15.06 \\
\hline HELQ & 0.07 & 1.11 & 6.75 & 6.60 & 1.84 & 31.60 & 3.61 & 1.82 & 17.69 & 0.36 & 2.15 & 13.08 \\
\hline XJWL & 0.50 & 2.43 & 13.17 & 6.41 & 1.46 & 30.29 & 1.36 & 1.68 & 18.82 & 1.15 & 2.38 & 13.76 \\
\hline Average & 0.27 & 1.78 & 9.71 & 5.95 & 2.01 & 28.75 & 2.78 & 1.99 & 17.98 & 1.08 & 2.67 & 13.97 \\
\hline
\end{tabular}


application, as they directly impact ionospheric TEC correlation characteristics.

\section{Model performance under different solar activities}

To investigate inter-day characteristics of model performance, statistical correction accuracy during parallel experimental periods (DOY 61-75 of 2014, 2016, and 2018) under different solar activities are analyzed and compared in below. The results are preliminary verification of the short-term accuracy and stability of the proposed algorithm.

\section{Statistical accuracy for each satellite}

The correction effect for each GPS satellite during the three experimental periods is calculated with different models, and Fig. 6 illustrates the results at station HELQ. We can see that the statistical performance of SIM is the best and very stable for every single GPS satellite. As shown by Fig. 6, the SIM bias is between 2-2 TECU without systematic offset, while the other products present systematic bias of several to 10 TECU. The STD of SIM and the other models is about 1-2 TECU and 2-3 TECU, respectively, under middle/low solar activities, and around
Fig. 6 Statistical STEC bias and STD for each GPS satellite during different experimental periods at user station HELQ. Performance results for SIM (top), UQRG (second), IOSR (third), and CRTC (bottom)
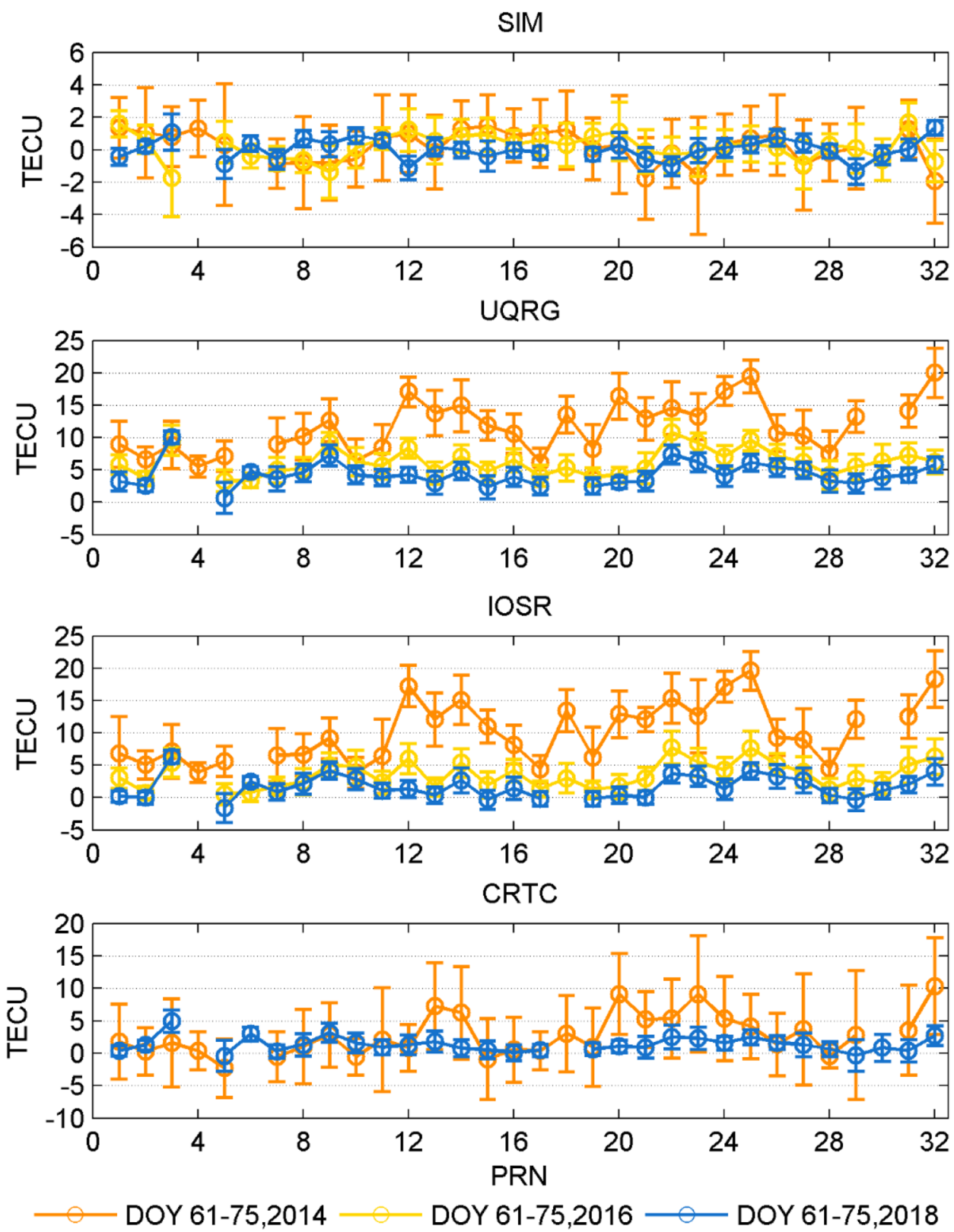
2-4 TECU and 6-10 TECU, respectively, under high solar activities.

\section{Statistical accuracy for all satellites}

The statistical performance of the four models for the GPS system is calculated and discussed below. The daily correction accuracy for all GPS satellites during experimental periods under different solar activities at station HELQ is shown in Fig. 7. The statistical results of different models are given in Fig. 8 for each period and user station.

According to the results in Fig. 7, SIM's STEC bias for all GPS satellites is constantly very small even under high solar activities, suggesting its good consistency with the regional/ local ionosphere. The SIM STD is also smaller than the other model corrections, meaning its performance is more stable than the other three VTEC models. As a result, the
SIM correction accuracy is remarkably improved than the GIM/RIM products.

We can know from the results in Fig. 8 that the correction accuracy of SIM is most satisfactory among the four ionospheric correction models. SIM's STEC bias is only by several decimal to centile TECU regardless of the solar activity level, which is better than other GIM/RIM models by 1-2 orders of magnitude. On each user station, the difference of STD between different models is relatively small, so the model performance is mainly determined by its systematic error. Generally, SIM can correct about $90 \%$ of ionospheric STEC RMS errors. Moreover, the performance of SIM is the best on station HELQ, followed by GSMA and XJWL. This indicates the importance of inter-station distance in the SIM algorithm. In comparison, UQRG can correct about 70-80\% of RMS errors. Its correction accuracy at GSMA is better than other user stations, and its performance can
Fig. 7 Daily STEC bias and STD (left) and relative RMS error (right) for all GPS satellites at station HELQ under different solar activities. Different model performances during DOY 61-75 in 2014 (top), DOY 61-75 in 2016 (middle), and DOY 61-75 in 2018 (bottom)
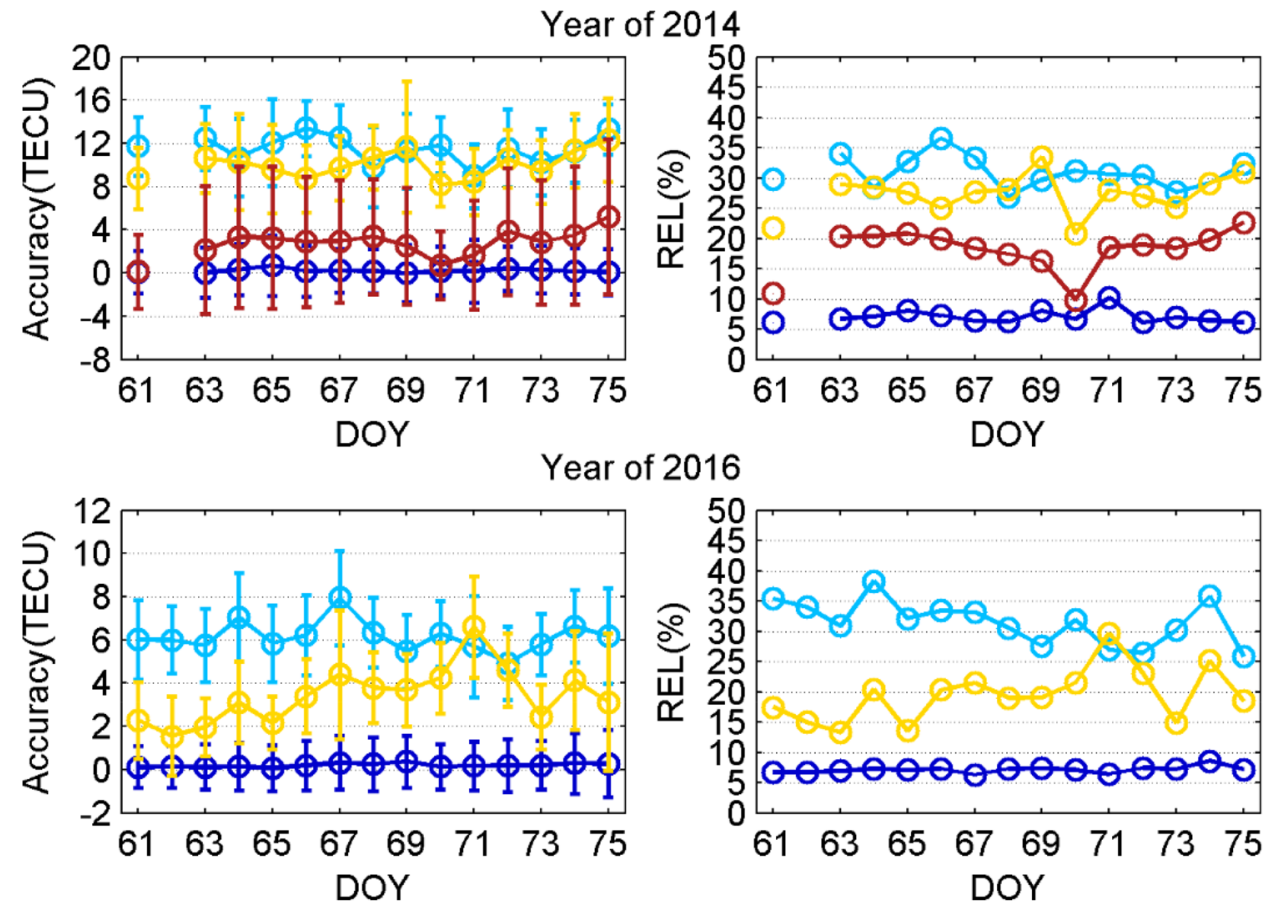

Year of 2018
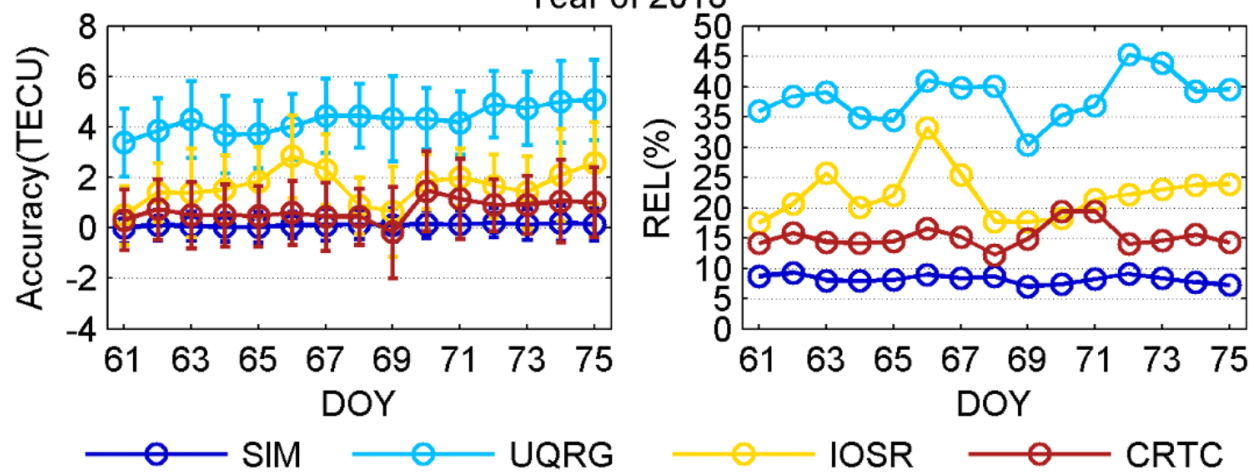


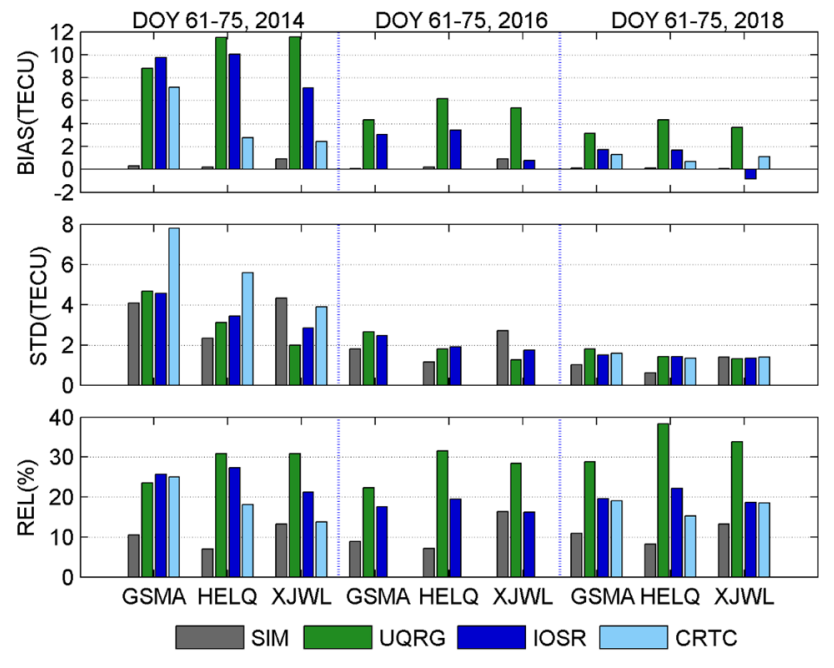

Fig. 8 Statistical correction accuracy for all GPS satellites for different models at each user station during different experimental periods and solar activities. STEC bias (top) results, STEC STD (middle), and STEC relative RMS error (bottom).

be equivalent to that of IOSR in some cases. In consideration of the number of reference stations used for modeling, UQRG proves to be quite well-performing. As RIM products of China, about $85 \%$ and $80 \%$ of RMS errors can be corrected by CRTC and IOSR, respectively. Although fewer domestic sites are used in CRTC, higher temporal resolution contributes to its accuracy improvement. But its STD appeared to be bigger in 2014 , this may be caused by the initial pilot period, and this problem is solved during the following updates to improve product stability.

\section{Seasonal model performance}

Statistical correction accuracy of the four ionospheric models on all user stations during different seasonal test periods in 2014 is computed and given in Table 5. As shown by the results, the STEC biases of SIM are all less than 0.5 TECU, which is better than the other products by one order of magnitude. The bias and STD results of SIM in autumn and winter are smaller than those in spring and summer, and the bias and STD results of the other three products decline from spring to winter. The RMS errors correction percentages of all products are highest in autumn.

\section{Conclusions}

Unlike VTEC correction models, we design and propose a GNSS ionospheric STEC modeling and correction method based on SIM. The new algorithm avoids the modeling error induced by the mapping function, which is usually more
Table 5 Model performance for test periods in different seasons of 2014. The unit for bias and STD is TECU, and that for relative RMS error (REL) is in percentile (\%)

\begin{tabular}{lllrlr}
\hline Season & Test Period & Ionospheric Model & \multicolumn{3}{c}{ Ionospheric STEC } \\
& & & \multicolumn{3}{c}{ correction accuracy } \\
\cline { 4 - 6 } \cline { 3 - 5 } Spring & \multirow{2}{*}{ DOY 61-75 } & SIM & 0.46 & 3.55 & 10.23 \\
& & UQRG & 10.61 & 3.25 & 28.42 \\
& & IOSR & 8.95 & 3.60 & 24.72 \\
& & CRTC & 4.11 & 5.76 & 18.97 \\
Summer & DOY 165-179 & SIM & 0.49 & 3.65 & 12.96 \\
& & UQRG & 8.16 & 3.70 & 29.34 \\
& & IOSR & 6.25 & 3.60 & 24.03 \\
& & CRTC & 1.39 & 4.71 & 17.00 \\
Autumn & DOY 259-273 & SIM & 0.15 & 3.00 & 9.45 \\
& & UQRG & 4.97 & 4.14 & 19.36 \\
& & IOSR & 5.11 & 3.60 & 18.68 \\
& & CRTC & 0.95 & 4.19 & 13.51 \\
Winter & DOY 349-363 & SIM & 0.25 & 2.74 & 14.12 \\
& & UQRG & 4.39 & 3.16 & 24.31 \\
& & IOSR & 3.37 & 3.20 & 21.31 \\
& & CRTC & 1.07 & 3.54 & 17.61 \\
\hline
\end{tabular}

serious in mid and low latitudes with higher levels of ionospheric activities.

A major motivation of our work is to use a few monitoring stations to provide accurate ionospheric correction for GNSS users within a regional/local network up to several hundred kilometers. As STEC values observed from a single satellite vary smoothly on a continuous observation arc within a small coverage, the ionospheric STEC correction model can be established satellite-by-satellite using a low-order polynomial function. To verify the performance of the proposed algorithm, validation tests are carried out with GNSS observation data from CMONOC in real-time processing mode, and its correction accuracy is compared with those of three commonly-used ionospheric GIM and RIM products. It can be concluded from the results that the proposed SIM method can accurately and stably represent regional/local STEC variation for each satellite without systematic offset under different solar activities.

The inter-station distance is an important factor that needs to be considered during network set-up since the STEC SIM algorithm is proposed based on the highly similar and homogenous features in ionospheric TEC spatial distribution and variation. With increasing distance, the TEC correlation between different locations weakens, and the low-order polynomial function may no longer be adequate to model the ionospheric delay satisfactorily. Consequently, the performance of SIM would deteriorate as the coverage area extends. We 
mainly propose the STEC SIM algorithm to solve the problem of accurate and efficient regional/local ionospheric STEC modeling. Therefore, the inter-station distance is suggested to be dozens to several hundred kilometers, which is feasible to realize in the context of various densely distributed ground-based GNSS networks. For instance, there are currently nearly 200 GNSS sites covering all provinces across China in CMONOC alone. In addition, there are also numerous provincial and municipal Continuously Operating Reference Stations (CORS) networks nationwide. Furthermore, validation experiments in this manuscript are not designed to show the optimal performance of SIM but are intended to test the acceptable range of inter-station distance with satisfactory correction accuracy. The performance of SIM would be certainly better with shorter inter-station distances, like tens or 100-200 km. The experiment results in this paper show that the SIM algorithm can generally correct about $90 \%$ of STEC RMS errors with an average interstation distance of about 400 to $600 \mathrm{~km}$. We recommend the inter-station distance should not be further enlarged to make the utmost of the advantages of SIM algorithm.

In summary, the proposed algorithm can be satisfactorily applied in high-accuracy ionospheric correction for regional and local GNSS networks within hundreds of kilometers. Additionally, in the next step, more validation tests will be carried out extensively in different latitudes and areas around the world. The performance of the proposed algorithm should also be tested in real positioning applications, such as real-time PPP and RTK.

Acknowledgements Sincere thanks to CMONOC for providing GNSS observation data, UPC for rapid GIM product, and CEA and CAS for RIM product of China. This work is supported by the Alliance of International Science Organizations (Grant No. ANSO-CR-KP-2020-12), National Key Research Program of China (No. 2017YFE0131400), the China Natural Science Funds (No. 42122026, 42074043, 42174038), National Center for Research and Development of Poland (decision no. DWM/PL-CHN/97/2019, WPC1/ARTEMIS/2019) and Youth Innovation Promotion Association of CAS

Data Availability The datasets generated during and/or analyzed during the current study are available from the corresponding author on reasonable request. The CAS ionospheric TEC and DCB products are publicly available from CAS FTP site ftp://ftp.gipp.org.cn/product/ionex/.

Open Access This article is licensed under a Creative Commons Attribution 4.0 International License, which permits use, sharing, adaptation, distribution and reproduction in any medium or format, as long as you give appropriate credit to the original author(s) and the source, provide a link to the Creative Commons licence, and indicate if changes were made. The images or other third party material in this article are included in the article's Creative Commons licence, unless indicated otherwise in a credit line to the material. If material is not included in the article's Creative Commons licence and your intended use is not permitted by statutory regulation or exceeds the permitted use, you will need to obtain permission directly from the copyright holder. To view a copy of this licence, visit http://creativecommons.org/licenses/by/4.0/.

\section{References}

Conker RS, El-Arini MB (2002) An ionospheric obliquity process responsive to line-of-sight azimuth and elevation. Radio Sci 37(6): $1-9$

Feltens J (1998) Chapman profile approach for 3-D global TEC representation, IGS presentation. In: Proceedings of the 1998 IGS analysis centers workshop, ESOC, Darmstadt, Germany, February 9-11,pp 285-297

Geng J, Meng X, Dodson AH, Ge M, Teferle FN (2010) Rapid reconvergences to ambiguity-fixed solutions in precise point positioning. J Geod 84(12):705-714

Hernández-Pajares M, Juan J, Sanz J, Orus R, Garcia-Rigo A, Feltens J, Komjathy A, Schaer S, Krankowski A (2009) The IGS VTEC maps: a reliable source of ionospheric information since 1998. J Geod 83(3):263-275

Hernández-Pajares M, Roma-Dollase D, Krankowski A, García-Rigo A, Orús-Pérez R (2017) Methodology and consistency of slant and vertical assessments for ionospheric electron content models. J Geod 91(12):1405-1414

IS-GPS (2004) Navstar GPS space segment/navigation user interfaces (ICD-GPS-200D), Revision D, ARINC engineering services, LLC, El Segundo, CA, USA

Juan JM, Sanz J, Hernández-Pajares M, Samson J, Tossaint M, AragonAngel A, Salazar D (2012) Wide Area RTK: a satellite navigation system based on precise real-time ionospheric modeling. Radio Sci 47(02):1-14

Klobuchar JA (1987) Ionospheric time-delay algorithm for single-frequency GPS users. IEEE Trans Aero Electron Syst 23(3):325-331

Lanyi GE, Roth T (1988) A comparison of mapped and measured total ionospheric electron content using global positioning system and beacon satellite observations. Radio Sci 23(4):483-492

Li W, Li Z, Wang N, Liu A, Wang X, Wang L, Yuan H (2020) Adaptation of the NeQuick2 model for GNSS wide-area ionospheric delay correction in China and the surrounding areas. Adv Space Res. https://doi.org/10.1016/j.asr.2020.11.016

Li Z, Fan L, Yuan Y, Verhagen S, Bakker P, Yuan H, Zhong S (2014a) Mitigation of ionospheric delay in GPS/BDS single frequency PPP: assessment and application. China satellite navigation conference (CSNC) 2014 proceedings, vol II. Springer, Berlin, Heidelberg, pp 477-499

Li Z, Wang N, Wang L, Liu A, Yuan H, Zhang K (2019) Regional ionospheric TEC modeling based on a two-layer spherical harmonic approximation for real-time single-frequency PPP. J Geod. https:// doi.org/10.1007/s00190-019-01275-5

Li Z, Yuan Y, Fan L, Huo X, Hsu H (2014b) Determination of the differential code bias for current BDS satellites. IEEE Trans Geosci Remote Sens 52(7):3968-3979

Li Z, Yuan Y, Li H, Ou J, Huo X (2012) Two-step method for the determination of the differential code biases of compass satellites. J Geod 86(11):1059-1076

Montenbruck O, González Rodríguez B (2019) NeQuick-G performance assessment for space applications. GPS Solut. https://doi. org/10.1007/s10291-019-0931-2

Mylnikova AA, Yasyukevich YV, Kunitsyn VE, Padokhin AM (2015) Variability of GPS/GLONASS differential code biases. Results in Physics 5:9-10

Santis AD, Torta JM, Lowes FJ (1999) Spherical cap harmonics revisited and their relationship to ordinary spherical harmonics. Phys Chem Earth (a) 24(11-12):935-941

Schaer S (1999) Mapping and predicting the earth's ionosphere using the global positioning system, Ph.D. dissertation, Astronomical Institutes, University of Bern, Berne, Switzerland 
Shi C, Gu S, Lou Y, Ge M (2012) An improved approach to model ionospheric delays for single-frequency precise point positioning. Adv Space Res 49(12):1698-1708

Sparks L, Komjathy A, Mannucci AJ (2004) Estimating SBAS ionospheric delays without grids: the conical domain approach. Proc. ION NTM 2004, Institute of Navigation, San Diego, CA, USA, January 26 - 28, pp 530-541

Wang N, Yuan Y, Li Z, Montenbruck O, Tan B (2016) Determination of differential code biases with multi-GNSS observations. J Geod 90(3):209-228

Wang L, Li Z, Ge M, Neitzel F, Wang X, Yuan H (2019) Investigation of the performance of real-time BDS-only precise point positioning using the IGS real-time service. GPS Solut 23(3):66. https:// doi.org/10.1007/s10291-019-0856-9

Wang N, Li Z, Duan B, Hugentobler U, Wang L (2020) GPS and GLONASS observable-specific code bias estimation: comparison of solutions from the IGS and MGEX networks. J Geod. https://doi. org/10.1007/s00190-020-01404-5

Yang Y, Mao Y, Sun B (2020) Basic performance and future developments of BeiDou global navigation satellite system. Satell Navig 1(1):1. https://doi.org/10.1186/s43020-019-0006-0

Yasyukevich YV, Mylnikova AA, Kunitsyn VE, Padokhin AM (2015) Influence of GPS/GLONASS differential code biases on the determination accuracy of the absolute total electron content in the ionosphere. Geomag Aeron 55(6):763-769

Yasyukevich Y, Mylnikova A, Vesnin A (2020) GNSS-based nonnegative absolute ionosphere total electron content, its spatial gradients, time derivatives and differential code biases: boundedvariable least-squares and taylor series. Sensors 20(19):5702. https://doi.org/10.3390/s20195702

Zhang B, Teunissen PJ, Yuan Y (2017) On the short-term temporal variations of GNSS receiver differential phase biases. J Geodesy 91(5):563-572

Publisher's Note Springer Nature remains neutral with regard to jurisdictional claims in published maps and institutional affiliations.

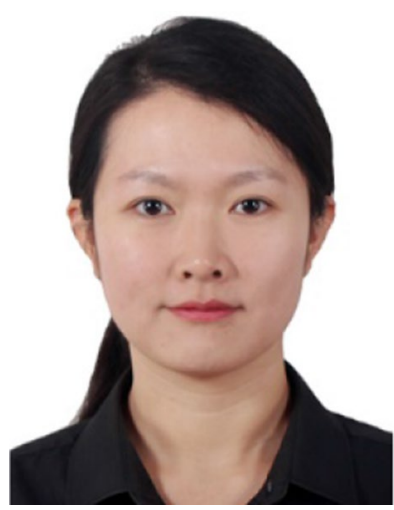

Wen Li is an engineer at Aerospace Information Research Institute (AIR), Chinese Academy of Sciences (CAS), and also a Ph.D. student at the University of Chinese Academy of Sciences (UCAS). She received a master's degree in geodesy and geomatics from Wuhan University in 2012. Her current researches are mainly focused on multi-GNSS ionospheric modeling and monitoring.

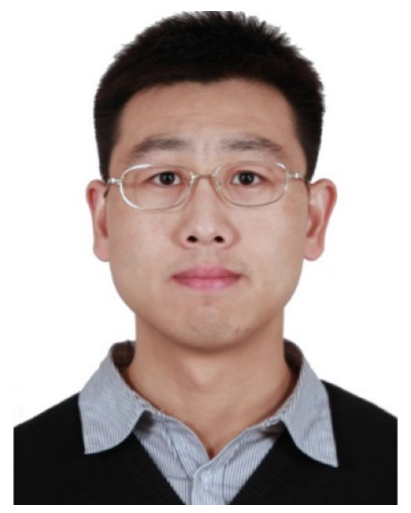

Zishen Li is an associate professor at AIR, CAS, and the research group leader on GNSS remote sensing and precise positioning. He received a Ph.D. degree in geodesy and surveying engineering from the Institute of Geodesy and Geophysics (IGG), CAS in 2013. His research activities involve real-time ionospheric remote sensing and correction for various GNSS applications.

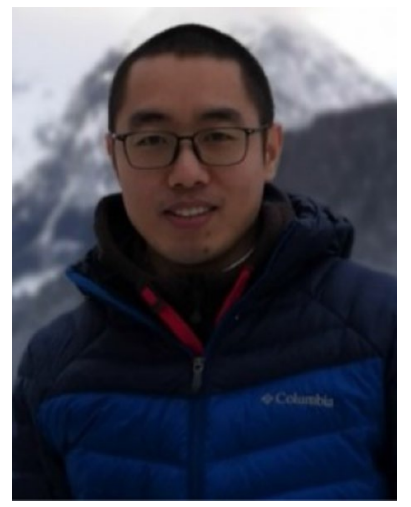

Ningbo Wang is an associate researcher at AIR, CAS. His work focuses on multi-GNSS bias handling and real-time ionospheric modeling using multiconstellation GNSS data.

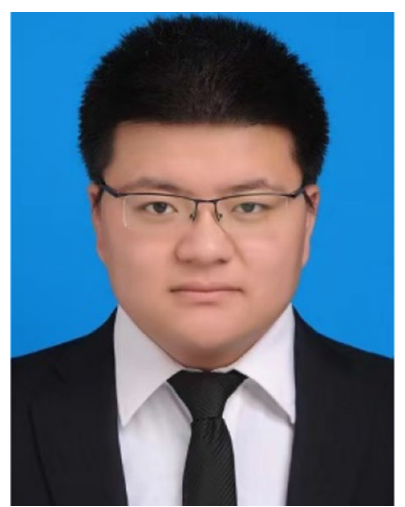

Ang Liu is a Ph.D. student at the University of Chinese Academy of Sciences (UCAS) and mainly engages in research on multiGNSS bias evaluation and estimation and ionospheric modeling through multi-source data fusion.

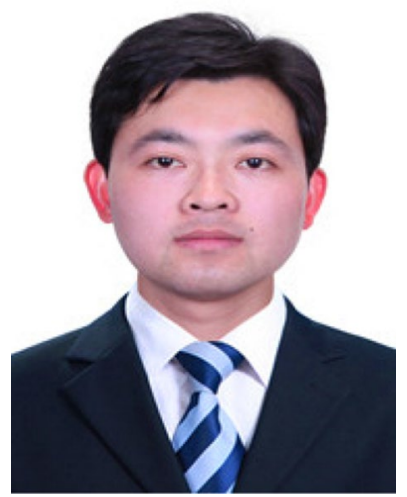

Kai Zhou is an engineer at AIR, CAS. He mainly works on threedimensional ionospheric modeling and the development of ionospheric monitoring equipment. 


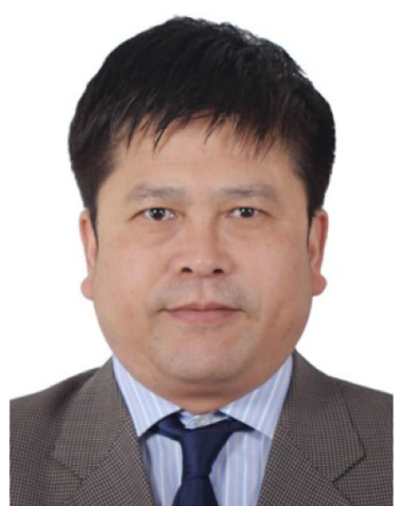

Hong Yuan is a professor and the director of the Navigation Department at AIR, CAS. He received a $\mathrm{Ph} . \mathrm{D}$. degree from Shanxi Astronomy Observatory, CAS, in 1995, and his research interests are GNSS ionospheric modeling and multi-sensorbased navigation.

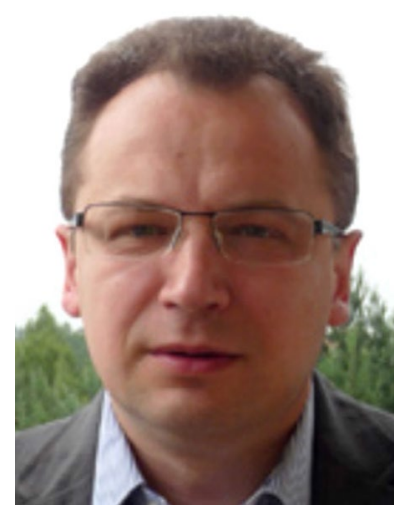

Andrzej Krankowski is a full professor at the Faculty of Geodesy, Geospatial and Civil Engineering (GGCE) of the University of Warmia and Mazury in Olsztyn (UWM), Poland and Head of Space Radio-Diagnostic Research Center (SRRC/UWM) and Lamkówko Satellite Observatory. His research interests include precise GNSS positioning and GNSS-based ionosphere modeling and forecasting for all GNSS users. 